\title{
Study on the Strategy of Acceleration Factor in Particle Swarm Optimization Algorithm
}

\author{
Zhe Li $^{1}$, Peng Bai ${ }^{1}$, R.-L. Tan ${ }^{1,2}$, Y.-B. Shang ${ }^{1}$, J.-J. Wang ${ }^{1}$ \\ ${ }^{1)}$ Research Center of Scientific Research Departments for Equipment development and Application, The University of Air Force \\ Engineering, Xi'an, Shanxi, China (kongyanshi@126.com) \\ 2) The University of Armed Police Engineering, Xi’an, Shaanxi, China (madamtan@126.com)
}

\begin{abstract}
Concerning the problem of Particle swarm optimization for solving complex multimodal function could easily fall into premature convergence, this paper proposed an algorithm of dynamically changing acceleration factor particle swarm optimization (CAPSO). According to the motion characteristics of the particles at different stages, acceleration factor of the particle velocity update formula were constructed as a monotonically increasing function and monotonically decreasing function. By setting the dynamic adjustment factor to dynamically determine the acceleration factor expression based on the actual simulation conditions, the algorithm had a better ability to adapt. Validated through four standard test functions and compared with other similar algorithms, numerical simulation results show that by adjusting the dynamic acceleration factor, the proposed algorithm had better optimization precision and execution ability compared with other algorithms.
\end{abstract}

Keywords - particle swarm optimization, acceleration factor, dynamic adjustment factor

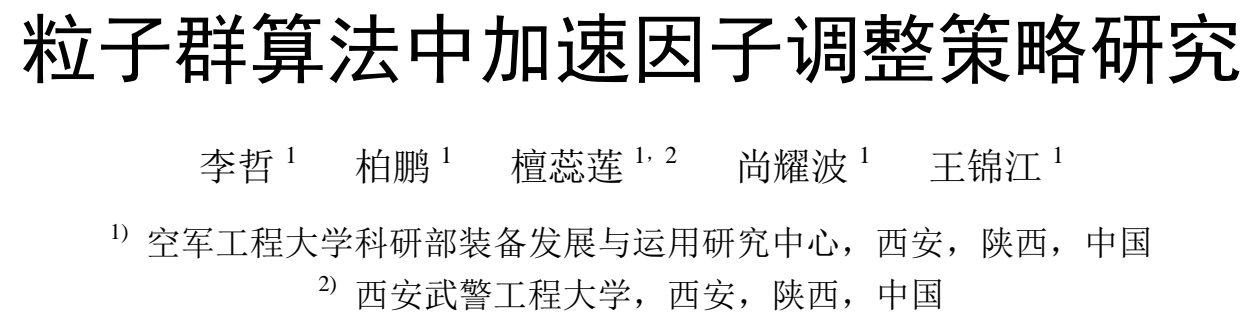

摘 要 针对粒子群算法在求解复杂多峰函数时极易陷入早熟收玫的问题, 提出一种动态改变加速因子的粒子群算法 (CAPSO)。 根据粒子在不同阶段的运动特性将粒子速度更新公式中的加速因子分别构造为单调递增函数和单调递减函数, 并设置动态调整因子根 据实际仿真条件动态确定加速因子的表达形式，从而使得算法具有更好的适应能力。通过 4 个标准测试函数进行验证，并与其他类似 算法进行比较的数值仿真结果表明, 通过调整动态加速因子, 所提算法具有更好的寻优精度和执行能力, 相比其他算法性能更优。

关键词 粒子群算法, 加速因子, 动态调整因子

1. 引言

粒子群算法（Particle Swarm Optimization，简称 PSO）收玫速度快、算法简单、所需参数少且易于实现, 因 此在函数优化、模式识别、系统辨识等领域得到广泛应用 $[1,2]$ 。然而, PSO 算法在演化过程中容易陷入局部最优, 研究表明, 通过参数调整可以在一定程度上提高粒子群算 法的整体性能 [3-6], Shi 和 Eberhart 最早引入了惯性权 重的概念 (SPSO) [4], 这也为后续学者在参数调整方面的 研究奠定了基础。然而, 大多数学者的研究主要集中在对
惯性因子的调整, 对加速因子的研究仍然处于起步阶段, 而且对于如何选取加速因子的问题仍无最优解决方案。

本文提出一种动态改变加速因子的粒子群算法 (CAPSO)。首先, 根据粒子的运动特点将加速因子分别构造 为单调递增函数和单调递减函数, 同时, 引入动态调整因 子对加速因子实时调整, 并从理论上分析了引入新参数后 算法的收玫性; 然后, 通过对已有惯性因子参数做出分析 对比, 选出最适合本算法的惯性因子; 最后, 通过 4 个具 有代表性的基准函数对新算法进行测试, 并与已有常用参 数调整算法进行对比。结果表明, 本文所提算法具有更好 
的寻优精度和执行性能, 在处理复杂多峰函数时亦能表现 出良好的性能。

\section{2. 标准粒子群算}

假设有 $\mathrm{m}$ 个粒子组成的种群 $X=\left(X_{1}, X_{2}, \cdots X_{m}\right)$, 则在一 个 D 维的搜索空间中, 第 $\mathrm{i}$ 个粒子在 D 维搜索空间的位置 可表示为向量 $X_{i}=\left(X_{i 1}, X_{i 2}, \cdots X_{i m}\right)^{T}$, 即问题的一个可能解。 若第 $\mathrm{i}$ 个粒子在 $\mathrm{D}$ 维空间的当前飞行速度为 $V_{i}=\left(V_{i 1}, V_{i 2}, \cdots V_{i D}\right)^{T}$, 个体极值为 $P_{i}=\left(P_{i 1}, P_{i 2}, \cdots P_{i D}\right)^{T}$, 种群的群 体极值为 $P_{g}=\left(P_{g 1}, P_{g 2}, \cdots P_{g D}\right)^{T}$, 则在每次迭代过程中, 粒子 将按公式 (1)（2）更新个体极值和群体极值从而更新自身 的速度和位置 $\left(P_{i}\right.$ 代表粒子 $\mathrm{i}$ 所经历的最好位置, $P_{g}$ 代表 种群的全局最优位置)。

$$
\begin{aligned}
V_{i d}^{n+1} & =\omega V_{i d}^{n}+c_{1} r_{1}\left(P_{i d}^{n}-X_{i d}^{n}\right)+c_{2} r_{2}\left(P_{g d}^{n}-X_{i d}^{n}\right) \\
X_{i d}^{n+1} & =X_{i d}^{n}+V_{i d}^{n+1}
\end{aligned}
$$

其中, $\omega$ 为惯性权重; $d=1,2 \ldots, D ; i=1,2 \ldots, n ; n$ 为 当前迭代次数; $V_{i d}{ }^{n}$ 为粒子的当前速度; $c_{1}$ 和 $c_{2}$ 称为加速因 子, 通常为非负的常数; $r_{1}$ 和 $r_{2}$ 为均匀分布的随机数, 分布 于 $[0,1]$ 之间; 为防止粒子的盲目搜索, 一般将其限制在 一定的区间 $\left[-X_{\text {max }}, X_{\text {max }}\right],\left[-V_{\text {max }}, V_{\text {max }}\right]$ 。

\section{2. 动态改变加速因子的 PSO 算法}

\subsection{PSO 算法中加速因子的选取}

目前对于 PSO 算法中加速因子 $\mathrm{c} 1, \mathrm{c} 2$ 的取值众说纷 纭, 学术界各学者的观点不一, 分歧很大, 大都根据自 己的实验结果的优劣而取的常数值, 主要学者给出的参 考值如表 1 所示。

表 1 加速因子常数参考值

\begin{tabular}{|c|c|}
\hline 学者代表人物 & $\mathrm{c} 1, \mathrm{c} 2$ 取值 \\
\hline Eberhart $^{[4]}$ & $\mathrm{c} 1=\mathrm{c} 2=1.49445$ \\
\hline Clerc $^{[7]}$ & $\mathrm{c} 1=\mathrm{c} 2=2.05$ \\
\hline Trelea $^{[8]}$ & $\mathrm{c} 1=\mathrm{c} 2=1.7$ \\
\hline
\end{tabular}

\section{2 改进的 PSO 算法}

Clerc 提出一种带收缩因子的粒子群算法 (CPSO) ${ }^{[5]}$, 一个简单的带收缩因子的粒子群算法定义为:

$$
\begin{gathered}
V_{i d}^{n+1}=\chi\left[V_{i d}^{n}+c_{1} r_{1}\left(P_{i d}^{n}-X_{i d}^{n}\right)+c_{2} r_{2}\left(P_{g d}^{n}-X_{i d}^{n}\right)\right] \\
\chi=\frac{2}{\left|2-l-\sqrt{l^{2}-4 l}\right|}, \quad l=c_{1}+c_{2}, \quad l>4
\end{gathered}
$$

其中 $l=4.1$, 虽然算法在收玫性方面有所改善, 但是 必须预先对算法进行限定, 否则在给定的迭代次数下很难 找到全局最优; 文献 [6] 提出了一种新的参数调整方法 ( VCPSO), 其速度更新公式中的加速因子 $c_{1}$ 和 $c_{2}$ 被构造成 了两个非线性递增的函数, 但是通过分析可知, $c_{1}$ 和 $c_{2}$ 主 要调节粒子 $P_{i}$ 和 $P_{g}$ 的最大步长, 表示粒子向个体极值和全 局极值的学习程度, 实际上我们期望的是在算法初期局部 搜索能力较强, 全局搜索能力较弱, 这样可以使得粒子的 搜索更精细, 先在较小范围内搜索局部最优值; 而在算法 后期我们则期望局部搜索能力变弱, 全局搜索能力增强, 这样粒子间交流加强, 获得的全局信息变多, 使得粒子能 在较广的区域内寻优, 从而避免粒子过早陷入局部最优而 无法跳出。基于这种思想, 本文对加速因子提出一种新的 调整策略, 将 $c_{1}$ 构造成为单调递减函数, $c_{2}$ 构造成为单调 递增函数, 其表达式如下:

$$
\begin{aligned}
& c_{1}(k)=\lambda_{1} *\left(1-e^{-\frac{K \max -k}{K \max }}\right) \\
& c_{2}(k)=\lambda_{2} *\left(1-e^{-\frac{k}{K \max }}\right)
\end{aligned}
$$

其中, $c_{1}(k)$ 和 $c_{2}(k)$ 即为新构造的加速因子函数表达 式, $K_{\text {max }}$ 为最大迭代次数, $k$ 为当前迭代次数, $\lambda_{i}$ 为动态 调整因子, 根据进化次数进行调整, 这样可以保证 $c_{i}(k)$ 的 极限值不超过各学者提出的经典值, 方便测试对比。通过 调整, 使得在迭代初期得到较大的 $c_{1}(k)$ 和较小的 $c_{2}(k)$, 个 体间交流较强; 在迭代后期得到较小的 $c_{1}(k)$ 和较大的 $c_{2}(k)$, 粒子间的全局合作能力增强。此时的速度更新公式 和位置更新公式为:

$$
\begin{gathered}
V_{i d}^{n+1}(k)=\omega(k) V_{i d}^{n}+c_{1}(k) r_{1}\left(P_{i d}^{n}-X_{i d}^{n}\right) \\
+c_{2}(k) r_{2}\left(P_{g d}^{n}-X_{i d}^{n}\right) \\
X_{i d}^{n+1}(k)=X_{i d}^{n}(k)+V_{i d}^{n+1}(k)
\end{gathered}
$$

\section{3 算法收敛性分析}

定理 1（PSO 算法收玫性能）当 $0<\omega<1$ 时, 若存在 $0<r_{1}+r_{2}<2(\omega+1)$, 则标准 PS0 算法收玫 ${ }^{[8]}$ 。

下面分析改进后粒子群算法的收敛性能:

定义: 令 $\gamma_{1}=c_{1}(k) r_{1}, \gamma_{2}=c_{2}(k) r_{2}$, 则公式 (7) 可变为:

$$
V_{i d}^{n+1}=\omega V_{i d}^{n}+\gamma_{1}\left(P_{i d}^{n}-X_{i d}^{n}\right)+\gamma_{2}\left(P_{g d}^{n}-X_{i d}^{n}\right)
$$

将公式 (9) 进一步分解得到

$$
V_{i d}^{n+1}=\omega V_{i d}^{n}+\gamma_{1} P_{i d}^{n}+\gamma_{2} P_{g d}^{n}-\left(\gamma_{1}+\gamma_{2}\right) X_{i d}^{n}
$$


令 $\gamma=\gamma_{1}+\gamma_{2}, \eta=\frac{\gamma_{1} p_{i d}{ }^{n+1}+\gamma_{2} p_{g d}{ }^{n+1}}{\gamma}$, 则公式 (9) 可写成:

$$
V_{i d}^{n+1}=\omega V_{i d}^{n}+\gamma\left(\eta-X_{i d}{ }^{n}\right)
$$

将公式（8）（11）改为差分方程形式表示为:

$$
\begin{aligned}
& V_{i d}^{n+1}(k+1)=\omega V_{i d}^{n}(k)+\gamma\left(\eta-X_{i d}{ }^{n}(k)\right) \\
& X_{i d}^{n+1}(k+1)=X_{i d}^{n}(k)+V_{i d}^{n+1}(k+1)
\end{aligned}
$$

令 $Y_{i d}{ }^{n}(k)=\eta-X_{i d}{ }^{n}(k)$, 则

$$
\begin{aligned}
& V_{i d}^{n+1}(k+1)=\omega V_{i d}^{n}(k)+\gamma Y_{i d}{ }^{n}(k) \\
& Y_{i d}{ }^{n+1}\left(k+1 \neq-\omega_{i d} V_{d}(k+)-\left(\gamma 1 \quad{ }_{i}{ }_{d}^{n} Y\right)\right.
\end{aligned}
$$

构造成矩阵的形式:

$$
\left[\begin{array}{l}
V_{i d}(k+1 \\
Y_{i d}(k+1)
\end{array}\right]^{n+1}=\left[\begin{array}{cc}
\omega & \gamma \\
-\omega & 1-\gamma
\end{array}\right]\left[\begin{array}{c}
V_{i d}(k) \\
Y_{i d}(k)
\end{array}\right]^{n}
$$

设 $P_{i d}(k)=\left[\begin{array}{c}V_{i d}(k) \\ Y_{i d}(k)\end{array}\right], \quad A=\left[\begin{array}{cc}\omega & \gamma \\ -\omega & 1-\gamma\end{array}\right]$, 则可以将上式改为:

$$
P_{i d}{ }^{n+1}(k+1)=A P_{i d}{ }^{n}(k)
$$

其中 $\mathrm{A}$ 矩阵的特征值为:

$$
\lambda_{1,2}=\frac{\omega+1-\gamma \pm \sqrt{(\omega+1-\gamma)^{2}-4 \omega}}{2}
$$

因此可得：

$$
\left[\begin{array}{c}
V_{i d}(k) \\
Y_{i d}(k)
\end{array}\right]^{n+1}=Q\left[\begin{array}{cc}
\lambda_{1} & 0 \\
0 & \lambda_{2}
\end{array}\right] Q^{-1}\left[\begin{array}{c}
V_{i d}(k) \\
Y_{i d}(k)
\end{array}\right]^{n}
$$

可知当且仅当 $\left|\lambda_{1,2}\right|<1$ 时公式收敛, 则 $\left|\frac{\omega+1-\gamma \pm \sqrt{(\omega+1-\gamma)^{2}-4 \omega}}{2}\right|<1$ 可以解得:

$$
\begin{aligned}
& 0<\gamma<2 \omega+2 \\
& 0<\omega<1
\end{aligned}
$$

因此改进后的算法也是是收敛的。

\section{4 算法实现}

Step1：初始化参数。设置惯性权重 $\omega$, 随机因子 $r 1$ 和 $\mathrm{r} 2$, 最大迭代次数 $\mathrm{K}_{\max }$, 初始速度 $V_{i d}$, 空间维数 $\mathrm{D}$, 并 在所定义的搜索空间中随机生成 $\mathrm{m}$ 个粒子组成初始种群。

Step2: 根据需求确定加速因子的取值范围, 从而计算
出相应的调整参数 $\lambda_{i}$, 最终确定 $c_{1}(k), c_{2}(k)$ 的表达式。

Step3: 计算种群中各粒子在每一维空间中的适应度函 数值, 根据公式（7）更新粒子速度，根据公式（8）更 新粒子空间位置, 迭代寻优, 从而产生新的种群, 实现个 体极值和群体极值的更新。

Step4：判断是否满足终止条件, 若满足, 则停止寻优; 否则转入 step3, 直到达到最大迭代次数才终止。

\section{3. 仿真实验及性能分析}

\section{1 测试函数}

为了测试算法的有效性，应用本文所提算法对 4 个 较常用于测试优化算法性能的基准函数进行测试, 各函 数名称与表达式如表 2 所示。

表 2 测试函数

\begin{tabular}{|c|c|c|}
\hline 函数 & 函数表达式 & 搜索空间 \\
\hline$f_{1}:$ Sphere & $\sum_{i=1}^{n} x_{i}^{2}$ & {$[-100,100]$} \\
\hline$f_{2}:$ Rosenbrock & $\sum_{i=1}^{n-1}\left[100\left(x_{i+1}-x_{i}^{2}\right)^{2}+\left(x_{i}-1\right)^{2}\right]$ & {$[-30,30]$} \\
\hline$f_{3}:$ Rastrigrin & $\sum_{i=1}^{n}\left[x_{i}^{2}-10 \cos \left(2 \pi x_{i}\right)+10\right]$ & {$[-5.12,5.12]$} \\
\hline$f_{4}:$ Griewank & $\frac{1}{4000} \sum_{i=1}^{n} x_{i}^{2}-\prod_{i=1}^{n} \cos \frac{x_{i}}{\sqrt{i}}+1$ & {$[-600,600]$} \\
\hline
\end{tabular}

3.2 不同算法之间的性能比较

设粒子种群规模为 20 ; 最大迭代次数为 300 ; 惯性 权重 $\omega(k)=\omega_{\text {start }}-\left(\omega_{\text {start }}-\omega_{\text {end }}\right)\left[\frac{2 k}{K_{\text {max }}}-\left(\frac{k}{K_{\text {max }}}\right)^{2}\right]$, 初始惯性权重 $\omega_{\text {start }}=0.9$, 迭代到最大次数时的惯性权重 $\omega_{\text {end }}=0.4, K_{\text {max }}$ 为 最大迭代次数; 每个基准测试函数独立在 SPSO 算法, CPSO 算法, VCPSO 算法, CAPSO 算法中各运行 300 次, 分别求出 最小适应度函数的最优值, 平均值, 最差值作为评价标准。 各测试基准函数所选取的参数如表 3 所示。如表 4 所示 为各基准函数在这四种算法中的测试结果对比。

表 3 算法参数

\begin{tabular}{|c|c|c|c|}
\hline 算 法 & $\omega$ & $c_{1}$ & $c_{2}$ \\
\hline SPSO & $\omega(k)$ & 1.49445 & 1.49445 \\
\hline CPSO & 0.729 & 1.49445 & 1.49445 \\
\hline VCPSO & $\omega(k)$ & $2 *\left(1-e^{-\frac{k}{K \max }}\right)$ & $2 * e^{-\frac{K \max -k}{K \max }}$ \\
\hline CAPSO & $\omega(k)$ & 公式 $(5)$ & 公式 $(6)$ \\
\hline
\end{tabular}


表 4 测试函数结果对比

\begin{tabular}{|c|c|c|c|c|}
\hline 函数 & 算 法 & 最优解 & 平均值 & 最差解 \\
\hline \multirow{6}{*}{$f_{1}$} & SPSO & $6.9530 \mathrm{e}-005$ & $1.5466 \mathrm{e}-004$ & 0.0016 \\
& CPSO & $2.1990 \mathrm{e}-004$ & $2.1995 \mathrm{e}-004$ & 0.0022 \\
& VCPSO & $1.9435 \mathrm{e}-004$ & $1.5000 \mathrm{e}-004$ & 0.0069 \\
& CAPSO & $2.3755 \mathrm{e}-005$ & $1.4570 \mathrm{e}-004$ & 0.0022 \\
\hline \multirow{5}{*}{$f_{2}$} & SPSO & 7.3097 & 7.4032 & 8.4264 \\
& CPSO & 9.8691 & 9.8691 & 9.8693 \\
& VCPSO & 9.0659 & 9.1450 & 9.3572 \\
& CAPSO & 8.7233 & 8.7371 & 8.7606 \\
\hline \multirow{5}{*}{$f_{3}$} & SPSO & 3.1081 & 4.4617 & 8.5962 \\
& CPSO & 5.9712 & 5.9712 & 5.9712 \\
& VCPSO & 4.0111 & 4.1311 & 4.8050 \\
& CAPSO & 3.9917 & 4.0035 & 4.0584 \\
\hline \multirow{6}{*}{$f_{4}$} & SPSO & $7.4988 \mathrm{e}-005$ & $1.3286 \mathrm{e}-004$ & $3.4349 \mathrm{e}-004$ \\
& CPSO & $3.0000 \mathrm{e}-004$ & $3.0000 \mathrm{e}-004$ & $3.0000 \mathrm{e}-004$ \\
& VCPSO & $8.9972 \mathrm{e}-005$ & $2.1157 \mathrm{e}-004$ & $6.6196 \mathrm{e}-004$ \\
& CAPSO & $5.9862 \mathrm{e}-005$ & $6.9927 \mathrm{e}-005$ & $1.7153 \mathrm{e}-004$ \\
\hline
\end{tabular}

由表 4 数据对比可以看出, 在对单峰函数 $f_{1}$ 的优化中, 本文算法在寻优精度方面取得了很好的效果。在最优解部 分, 测试结果与 SPSO 算法测试结果相近, 但是比 CPSO 算 法和 VCPSO 算法的最优解低了一个数量级, 在平均值方面 也是最接近理论最值的, 具有更好的寻优精度; 在对测试 函数 $f_{2}$ 的优化中, 本文算法在最优解、平均值和最差解之 间的差距最小，具有较好的执行性能; 在对测试函数 $f_{3}$ 的 优化中, 本文算法最优解和最差解差距最小, 仅为 0.0667 , 说明该算法局部寻优能力最强; 在对测试函数 $f_{4}$ 的优化中, 本文算法的优化效果较 SPSO 算法和 CPSO 算法略显逊色, 但是比 VCPSO 强, 总体而言, 在处理复杂多峰函数时, 离 理论值也比较近，达到了预期优化目的。

\section{4. 结语}

加速因子的变化会影响算法的收玫性能, 小的加速因 子可以使粒子在远离目标区域震荡, 大的加速因子可以使 粒子迅速向目标区域移动, 根据粒子不同阶段的特性选取
合适的加速因子可以使得粒子群算法具有更好的寻优能 力。本文通过对加速因子的研究提出了一种动态改变加速 因子的粒子群算法, 根据粒子的运动特点将加速因子构造 为单调函数表示形式，引入动态调整因子 $\lambda_{i}$ 根据实际测试 需要最终确定加速因子的表达形式，从而改进了算法的性 能。与已有算法进行比较的结果表明, 本文所提算法能够 很好处理复杂多峰函数, 具有更好的寻优精度和执行性能。

\section{参考文献(References)}

[1] Perez R E, Behdinan K. Particle swarm approach for structural design optimization. Computers \& Structures, 2007, 85(19/20): 1579-1588.

[2] Coelho L S, Sierakowski C A. A software tool for teaching of particle swarm optimization fundamentals. Advances in Engineering Software, 2008, 39(11): 877-887.

[3] Jiao B, Lian Z G, Gu X S. A dynamic inertia weight particle swarm optimization algorithm. Chaos Solitons \& Fractals, 2008, 37(3): 698-705.

[4] Shi Y, Eberhart R C. A modified particle swarm optimizer. Proceedings of IEEE Enternational Conference on Evolutionary Computation, Anchorage, 1998, 69-73.

[5] Clerc M. The swarm and the queen: Towards a deterministic and adaptive particle swarm optimization. Proceedings of the Congress of Evolutionary Computation, Washington, 1999: 1951-1957.

[6] RenWeijian, TaoLin. Research on Pump-jack Fault Diagnosis Method Based on Particle Swarm Optimization. Journal of System Simulation, 2012, 24(2):482-492.

[7] Clerc M. The swarm and the queen: Towards a deterministic and adaptive particle swarm optimization. IEEE Transactions on Evolutionary Computation, Washington, 2002:58-73.

[8] Trelea I C. The particle swarm-explosion, stability, and convergence in a multidimensional complex space: optimization algorithm: Convergence analysis and parameter selection; Information Process Letters, 2003, 85(6):317-325 